\title{
Diferenciando grados de sofisticación epistémica durante la resolución de un problema de electrostática
}

\author{
Differentiating epistemic sophistication degrees \\ during the process of solving an electrostatic problem
}

Andrea Perea ${ }^{1}$. Laura Buteler ${ }^{2}$. Enrique Coleoni ${ }^{2}$

\begin{abstract}
Resumen: El presente es un estudio en el cual se analizan las producciones de tres estudiantes de un curso universitario introductorio mientras resuelven un problema de electrostática. El objeto de estudio son las epistemologías personales, las cuales sirven a los estudiantes, y a los sujetos en general, para gestionar el propio conocimiento y posicionarse de maneras particulares frente a éste durante tareas cognitivas como la resolución de problemas. Se presenta un aporte de carácter teórico en relación al constructo de sofisticación epistémica. Se proveen elementos que permiten graduarla y tomarla como un indicador de la experticia de los sujetos en el plano epistémico. La importancia de los resultados radica en la posibilidad de visibilizar más la dimensión epistémica en el aprendizaje de la física.
\end{abstract}

Palabras clave: Enseñanza de la física. Enseñanza superior. Epistemología personal. Resolución de problemas. Electrostática.

\begin{abstract}
The present paper presents a case study in which an analysis is carried out on the production of three introductory physics students while solving an electrostatic problem at college level. The objects under study are students' personal epistemologies, which enable them, and everyone, to deal with their own knowledge in different ways when confronted to cognitive activities such as problem solving. The contribution of this paper is theoretical, and is related to the construct of epistemic sophistication. Our analysis provides elements that allow for a grading of sophistication as an indicator of subjects' epistemic expertise. Results are important in their potential value to better understand the epistemic dimension in the learning of Physics.
\end{abstract}

Keywords: Physics teaching. Higher education. Personal epistemology. Problem solving. Electrostatics.

\footnotetext{
${ }^{1}$ Universidad Nacional de Córdoba, Córdoba, Argentina. E-mail: <andreaperea77@gmail.com>.

${ }^{2}$ Universidad Nacional de Córdoba, Facultad de Matemática, Astronomía y Física, Córdoba, Argentina.
} 


\section{Introducción}

Las concepciones epistemológicas de los estudiantes influyen en los resultados de su aprendizaje y constituyen un factor que interfiere en dicho proceso (CAMPANARIO; OTERO, 2000; LISING; ELBY, 2005). Sin embargo, el término "concepciones epistemológicas" es polisémico. Algunas investigaciones entienden que éstas se refieren a las ideas o creencias que los estudiantes tienen en relación a cómo la comunidad científica construye y valida el conocimiento (por ejemplo, GURIDI, 1999; WAINMAIER, 2003; WAINMAIER; SPELTINI; SALINAS, 2011). Desde esta perspectiva, el término "concepciones epistemológicas" se aplica para hacer referencia a las nociones de los estudiantes sobre la naturaleza de las ciencias y del conocimiento científico. Denominaremos esta acepción del término como epistemologías públicas o formales.

Sandoval (2005) realiza una revisión exhaustiva sobre investigaciones realizadas en relación a las creencias de los estudiantes sobre la naturaleza de las ciencias (NOS, por su siglas en inglés, Nature of Science). Argumenta que los estudios sobre epistemologías públicas tendrían que extender el análisis a la práctica y relevar también los objetivos y criterios que los estudiantes emplean durante sus propios procesos de indagación, tendiendo así un puente entre las epistemologías públicas y las epistemologías prácticas de los estudiantes. Sandoval (2005) remarca claramente la diferencia entre tales epistemologías. Las epistemologías prácticas corresponden al conjunto de ideas que poseen los estudiantes sobre su propia producción de conocimiento escolar, son las creencias epistemológicas que guían su práctica. Las epistemologías públicas se refieren al conjunto de ideas sobre el conocimiento científico público y su producción: son las ideas que los estudiantes parecen tener sobre la ciencia profesional. Sandoval considera que para la mayoría de las personas, las epistemologías prácticas no son coherentes, y son mayoritariamente tácitas. Las mismas se ven reflejadas en las decisiones epistémicas que las personas toman durante su propia construcción y evaluación del conocimiento científico. Este autor indica que la capacidad de indagación de los estudiantes está guiada por las epistemologías prácticas de los mismos.

En algunos de sus trabajos Elby y Hammer (2001) y Hammer y Elby (2002) se enfocan en estudiar las llamadas epistemologías personales de los estudiantes que se corresponden con las que Sandoval (2005) llama epistemologías prácticas. Estas epistemologías personales son justamente las que permiten a las personas establecer (ya sea implícita o explícitamente) cuál es el conocimiento adecuado para abordar una situación particular. Dicho de otro modo, las epistemologías personales son las que habilitan al estudiante a dar respuesta a la pregunta tácita: ¿de qué se trata esta actividad? ¿Qué se supone que tengo que hacer acá? Esta perspectiva personal de las epistemologías posibilita un mejor entendimiento de las tareas cognitivas que ocurren durante el aprendizaje, tales como la argumentación y la resolución de problemas.

Elby y Hammer (2001) remarcan que es importante tener en cuenta que las epistemologías personales productivas - las que ayudan al estudiante a aprender - a veces difieren de las concepciones epistemológicas públicas expuestas por filósofos y científicos sociales. Una epistemología es productiva si genera comportamientos, actitudes y/o hábitos que llevan a las personas a superar obstáculos durante su aprendizaje. Dichos autores sugieren que muchas de las epistemologías personales ingenuas consisten en recursos epistemológicos o epistémicos -frecuentemente implícitos, a menudo inarticulados - que pueden ser activados en diferentes combinaciones por diferentes contextos. Mencionan que la investigación debería enfocarse también en 
identificar los recursos epistemológicos que los estudiantes usan en algunos contextos, pero no los aplican en otros en donde también podrían ser útiles. El docente avezado en estas ideas podría ayudar a sus estudiantes a "reorganizar" el conocimiento epistemológico que ellos ya poseen. Un abordaje como el que se plantea, en el cual las epistemologías personales resultan de la activación de recursos, plantea algunas ventajas interesantes. En primer lugar, permite entender naturalmente las diferencias entre los comportamientos de sujetos que han sido instruidos de la misma manera (aun cuando pertenezcan al mismo curso). Al mismo tiempo, puede explicar mejor la dependencia contextual en la sustancia de las epistemologías ingenuas y sofisticadas. La activación de un determinado conjunto de recursos es un proceso idiosincrático y contextual. Ciertas características del contexto pueden promover la activación de ciertos recursos en un sujeto y no en otros.

Lising y Elby (2005) proponen que las epistemologías públicas (o formales) y las epistemologías personales (o prácticas) de un estudiante pueden diferir entre sí significativamente. Por ejemplo, un estudiante puede dudar de la coherencia de su propio conocimiento (epistemología personal), pero puede esperar que los científicos busquen y encuentren coherencia (epistemología pública). Hogan (1999) encontró que la epistemología personal está fuertemente vinculada al comportamiento del estudiante, mientras que la epistemología pública prácticamente no manifiesta ningún efecto. Estos resultados apuntan a que las epistemologías personales son más importantes para el aprendizaje que lo que lo son las epistemologías públicas, lo cual despierta un mayor interés para centrarse en el estudio de las primeras.

En este trabajo nos proponemos avanzar en la comprensión de cómo las epistemologías personales se ponen en evidencia durante la resolución de problemas y permiten a los estudiantes gestionar su conocimiento. Este estudio forma parte de uno más amplio que pretende develar cómo y cuándo los estudiantes de física desarrollan la dimensión epistémica en temas de electrostática durante la resolución de problemas, durante el período en el cual asisten a clases de un curso cuatrimestral de electricidad y magnetismo.

\section{Marco teórico}

El marco teórico que se utiliza para analizar las epistemologías personales de los estudiantes es el marco de los Recursos Cognitivos. Éste se basa en una combinación de resultados básicos seleccionados de la investigación educativa, de la neurociencia, y de la ciencia del comportamiento. Proporciona ontologías - clases de elementos estructurales y la manera en que estos se comportan - lo que permite un rango de estructuras posibles e interacciones construidas desde esos elementos. El marco de los recursos es un modelo tipo red, asociativo, con estructuras de control, y enlaces dinámicos (BING; REDISH, 2009).

Pensar en términos de elementos cognitivos múltiples permite modelar el pensamiento de manera que pueda responder diferente en distintos momentos. La variabilidad de los recursos no es al azar, es decir, no se activan o desactivan aleatoriamente. Esta variación es mejor caracterizada como entre múltiples coherencias (HAMMER et al., 2005).

Algunos de los componentes significativos de dicha estructura de control son los llamados recursos epistemológicos o epistémicos. Un recurso epistémico es un elemento que representa un paquete de información fuertemente agrupado que, cuando es activado, lleva al individuo a 
interpretar el conocimiento en cuestión bajo una cierta lente. Estos son dinámicos, es decir, que pueden ser activados y desactivados de un momento a otro. Varios autores (DISESSA, 1993; REDISH, 2004, entre otros) han afirmado que las posturas epistémicas son múltiples y muy sensibles al contexto. Una prueba más de la naturaleza múltiple de las epistemologías de los estudiantes es la desconexión que suele haber entre cómo los estudiantes ven a la naturaleza de la ciencia formal y cómo proceden para interpretar su propio trabajo en las clases de ciencias.

Un recurso epistémico particular puede ser útil en ciertos contextos y contraproducente en otros. Desde esta perspectiva, los estudiantes entienden el conocimiento de manera diferente en distintas situaciones. Diferentes temas, diferentes configuraciones de clase, e incluso diferentes actividades pueden activar distintos conjuntos de recursos. La estabilidad de la postura epistémica de un individuo puede depender del contexto social o material (HAMMER et al., 2005), y por lo tanto puede ser erróneo atribuir a un individuo, una postura particular (ROSENBERG; HAMMER; PHELAN, 2006).

Se pueden encontrar múltiples evidencias de la dependencia contextual de las posturas epistémicas. Por ejemplo, un alumno que trabaja sobre un problema de física puede pasar tiempo tratando de construir una historia explicativa sobre por qué se obtiene cierto resultado - trata al conocimiento como una construcción personal. Luego puede cambiar y buscar, ya sea en un libro de texto o en sus apuntes de clase, una regla pertinente para esta cuestión - trata al conocimiento como algo que proviene de la autoridad (BING; REDISH, 2009). Hammer (2004), Hammer et al. (2005) y Coleoni, Buteler y Perea (2014), presentan detallados ejemplos sobre algunos cambios que se dan en el razonamiento de los estudiantes mientras discuten y tratan de resolver la situación planteada por los investigadores.

Este modelo del pensamiento de los estudiantes incluye además un proceso por el cual el conjunto de todas las opciones posibles (conceptuales y epistemológicas) son reducidas a un tamaño manejable para ser consideradas por el individuo. Dicho proceso se llama enmarque. De acuerdo con Bing y Redish (2009), enmarcar se corresponde con la elección de recursos (usualmente inconsciente) que la mente hace para responder a la pregunta: ¿qué clase de actividad es la que tengo acá? O ¿cómo se supone que debo proceder en esta situación? En el plano fenomenológico, cada enmarque presenta una característica saliente que se corresponde con un recurso en particular: un recurso que domina el enmarque.

Para entender cómo estudiantes universitarios se responden (casi siempre implícitamente) la pregunta anterior mientras resuelven problemas de física que involucran razonamientos matemáticos, Bing y Redish (2009) propusieron una taxonomía tentativa para los enmarques epistémicos activados por esos estudiantes. Esos enmarques, y sus correspondientes recursos epistémicos dominantes, son los siguientes: Cálculo, Mapeo fisico, Invocación a la autoridad y Consistencia matemática. (Ver cuadro 1).

Enmarcar es un proceso cognitivo dinámico. La mente de la persona hace un juicio inicial de acuerdo con la naturaleza de la situación en cuestión, pero ese juicio es continuamente actualizado y reevaluado (BING; REDISH, 2009). Una persona no puede tener en cuenta de forma activa todo su conocimiento en una situación dada. Algún subconjunto es seleccionado en base a lo que la mente interpreta (generalmente muy rápido) sobre qué clase de situación se trata. Distintos individuos pueden enmarcar la misma información recibida de diferentes maneras (BING; REDISH, 2009). Todo el tiempo llega nueva información al estudiante ya sea en forma de un comentario de un compañero de clase, de una intervención del entrevistador, al 
dar vuelta la página de un libro, o incluso de asociaciones espontáneas realizadas en su propio cerebro. Esta nueva información puede llevar al estudiante a re enmarcar su actividad. Cuando enmarcan y re enmarcan su actividad, se activan y desactivan diferentes conjuntos de recursos. A veces los enmarques de los estudiantes pueden mostrar una considerable resistencia al cambio.

En el siguiente cuadro se muestran los cuatro enmarques mencionados por Bing y Redish (2009), y los indicadores primarios y secundarios que nos ayudan a identificarlos.

Cuadro 1: Cuatro enmarques conceptualizados por Bing y Redish (2009) y sus indicadores

\begin{tabular}{|c|c|c|c|c|}
\hline & Cálculo & Mapeo Físico & $\begin{array}{l}\text { Invocación de la } \\
\text { autoridad }\end{array}$ & $\begin{array}{c}\text { Consistencia } \\
\text { matemática }\end{array}$ \\
\hline $\begin{array}{l}\text { Clase de } \\
\text { argumento } \\
\text { usado }\end{array}$ & $\begin{array}{l}\text { Seguir } \\
\text { correctamente } \\
\text { pasos algorítmicos } \\
\text { lleva a un resultado } \\
\text { confiable. }\end{array}$ & $\begin{array}{l}\text { El buen ajuste } \\
\text { que existe entre } \\
\text { las observaciones } \\
\text { matemáticas y físicas } \\
\text { da fe del resultado. }\end{array}$ & $\begin{array}{l}\text { La afirmación por } \\
\text { parte de la autoridad } \\
\text { de un resultado o } \\
\text { de una regla le da } \\
\text { credibilidad. }\end{array}$ & $\begin{array}{l}\text { La similitud } \\
\text { o la conexión } \\
\text { lógica con otra } \\
\text { idea matemática } \\
\text { ofrecen validación. }\end{array}$ \\
\hline \multirow{3}{*}{$\begin{array}{l}\text { Otros } \\
\text { indicadores } \\
\text { comunes }\end{array}$} & $\begin{array}{l}\text { Enfocar en la } \\
\text { exactitud técnica. }\end{array}$ & $\begin{array}{l}\text { Explicación a partir } \\
\text { de un diagrama. }\end{array}$ & Citar una regla & $\begin{array}{l}\text { Analogía con otra } \\
\text { idea matemática. }\end{array}$ \\
\hline & \multirow{2}{*}{$\begin{array}{l}\text { Encadenamiento } \\
\text { matemático: se } \\
\text { necesita esto para } \\
\text { obtener aquello. }\end{array}$} & $\begin{array}{l}\text { Gestos } \\
\text { demostrativos. }\end{array}$ & $\begin{array}{l}\text { Ausencia de } \\
\text { razonamiento } \\
\text { encadenado. }\end{array}$ & \multirow[t]{2}{*}{ Categorización. } \\
\hline & & $\begin{array}{l}\text { Razonamiento } \\
\text { encadenado. }\end{array}$ & $\begin{array}{l}\text { Poco reconocimiento } \\
\text { de la subestructura. }\end{array}$ & \\
\hline
\end{tabular}

Fuente: Bing y Redish (2009).

Los estudiantes que tienen visiones epistémicas sofisticadas tienden a abordar al aprendizaje de manera más activa y a alcanzar una mejor comprensión conceptual (por ejemplo, LOUCA et al., 2004). Sin embargo, son escasos los estudios que definen la sofisticación epistémica. Bing y Redish (2009) conjeturan que las posturas epistémicas evolucionan de maneras complejas, en sentido de tiempo promedio, mientras los estudiantes avanzan en su educación. Los autores suponen que a medida que los estudiantes se vuelven más sofisticados y expertos, se vuelven más flexibles en sus enmarques y aumenta la creación de enmarques híbridos (combinaciones de varios). En otro de sus trabajos (BING; REDISH, 2012), se proponen dilucidar qué es la sofisticación epistémica, cómo se "revela" durante el proceso de resolución de un problema de física. En ese estudio, los autores diferencian básicamente 3 grados diferentes de sofisticación. En primer lugar, y como evidencia de nula sofisticación, están aquellos casos en los que los estudiantes no pueden cambiar de marco epistémico. Como ejemplo de un primer grado de sofisticación, no nulo, los autores hablan de flexibilidad epistémica. La flexibilidad epistémica es la posibilidad de enmarcar la tarea en más de una manera. Es un primer grado de sofisticación de la manera en que el sujeto puede responder a la pregunta (implícita) "qué debo hacer frente a esta tarea". Además de un primer enmarque para dar respuesta a esta pregunta, se evidencia la capacidad del sujeto de hacerlo mediante otro enmarque. Los autores muestran un ejemplo en el cual un sujeto (al que llaman $\mathrm{S}_{2}$ ) enmarca la tarea como Invocación a la Autoridad, 
pero en algún momento puede también enmarcar la misma como Mapeo Físico o Consistencia Matemática. Como ejemplo de un grado superior de sofisticación, los autores reportan el caso de un sujeto que además de evidenciar flexibilidad epistémica, muestra la capacidad de anidar nuevos enmarques en el enmarque inicial. En el caso reportado, la anidación es mostrada en el ejemplo del sujeto $\mathrm{S}_{3}$ quien no solamente tiene la flexibilidad de realizar diferentes enmarques sino que además puede anidarlos en un enmarque general al que los autores denominan de "valoración de coherencia".

El estudio que se presenta aquí analiza un caso de tres estudiantes universitarios resolviendo un problema de electrostática (enunciado 1), en el que se advierte un grado de sofisticación intermedio entre los dos (no nulos) reportados por Bing y Redish (2012). En particular, veremos que en el caso que se discutirá, los estudiantes pueden anidar nuevos enmarques en un enmarque incial, aunque no muestran la valoración de coherencia que implica la comparación de razonamientos producidos en cada uno de ellos. El hallazgo es importante, porque permite refinar la conceptualización de sofisticación epistémica, y avanzar hacia un esquema de análisis que permitirá una mejor diferenciación entre distintos grados de sofisticación y podrá ser útil para entender mejor cómo cambia la misma durante algunos trayectos educativos. Una búsqueda que es relevante para la investigación educativa y para la educación, porque pone en evidencia cuán oculta está la dimensión epistémica en los currículos de física, y porque ofrece algunos indicios para incorporarla explícitamente. Este trabajo se enmarca en otro mayor, que pretende estudiar, esas formas complejas de cambios en la dimensión epistémica durante trayectos educativos en física. El estudio que se presenta constituye un eslabón esencial en esa búsqueda. Sus resultados, que se consideran tentativos, serán sucesivamente revaluados y refinados en futuras instancias de análisis sobre un conjunto mayor de datos.

\section{El estudio}

a) Los sujetos. Los participantes de esta investigación son tres estudiantes ( $M, S$ y A) de segundo año de una licenciatura en física de una universidad pública de Argentina. Dichos estudiantes participaron en tres entrevistas en las que resolvieron un conjunto de problemas de electrostática durante el cuarto cuatrimestre de la carrera en el año 2012. En ese cuatrimestre se encontraban cursando la asignatura Física General III, que involucra contenidos sobre electrostática, electrodinámica clásica, magnetismo y electromagnetismo clásico. Tales contenidos se enseñan al nivel de los presentados en libros como el de Serway (1997) y el de Resnick, Halliday y Krane (1993).Las clases del curso tienen una frecuencia de dos encuentros semanales. Cada uno de ellos consiste en una clase teórica expositiva (2 h) y una clase práctica de problemas (2 h). Las entrevistas fueron llevadas a cabo por la primera autora de este estudio, y ninguno de los autores formó parte del equipo docente de esta asignatura para estos estudiantes.

Los estudiantes que participaron de las entrevistas lo hicieron de manera voluntaria, luego de que el grupo de investigación realizara una convocatoria a todo el grupo clase de Física III. Estos tres alumnos tenían, al momento de las entrevistas, un desempeño académico promedio representativo del grupo de estudiantes de ese curso. El promedio histórico de la carrera de licenciatura de física es 7 y la duración promedio de la carrera es de 6 años, siendo 5 lo que estipula el plan de estudios. En lo que respecta a cada uno de los participantes, al 
momento de entrevistarlos durante el segundo cuatrimestre de segundo año, podemos decir lo siguiente: M: Había aprobado 5 materias de las 10 que contempla el plan de estudios hasta ese momento, con un promedio de 5,6. S: Había aprobado 6 de las 10 materias que contempla el plan de estudios hasta ese momento, con un promedio de 6,17. A: Había aprobado 7 de las 10 materias, con un promedio de 7,43.

b) La tarea. Se realizaron tres entrevistas grupales a los tres estudiantes alrededor de una tarea que contenía un conjunto de problemas. En este estudio se reportan resultados de la segunda entrevista en relación a un problema. La consigna de la entrevistadora (referida como $\mathbf{E}$ en los diálogos) era que resolvieran el problema entre todos. Sus intervenciones se limitaban a promover la discusión, exponer los puntos de vista de los entrevistados, o realizar preguntas para entender mejor las ideas surgidas. Las entrevistas fueron registradas en formato audio-visual.

Se utiliza la resolución de problemas como tarea, dado que entendemos que las epistemologías personales se ponen en evidencia mientras abordan una práctica de clase, y una práctica muy habitual en los cursos de física es la resolución de problemas. La elección del problema no es arbitraria: a fin de generar verbalizaciones extensas que nos permitan "capturar" los razonamientos de los estudiantes, se eligió un problema que promueva el análisis cualitativo por sobre los cálculos.

\section{Enunciado 1: el problema utilizado}

\section{Problema 1.}

Una esfera sólida conductora descargada posee en su interior una cavidad concéntrica (también esférica).

a) ¿'Tiene alguna influencia el conductor sobre el campo eléctrico exterior a él generado por una carga puntual $+Q$ ubicada en el centro de su cavidad?

b) Si la carga puntual ubicada en la cavidad no se encontrara justo en el centro de la misma ¿cuáles serían las respuestas del punto anterior?

En el punto (a), al estar centrada la carga puntual +Q en el interior de la esfera hueca conductora, se produce una redistribución homogénea de cargas en el conductor. Tal redistribución sucede de manera tal que en la pared interior de la esfera aparece una carga neta-Q distribuida homogéneamente (atraída por la carga puntual $+Q$ ), mientras que en la pared exterior aparece una carga neta $+Q$ también distribuida homogéneamente (repelida por la carga puntual $+Q$ ), manteniendo de esta manera la nulidad del campo eléctrico en el interior del conductor. En el punto (b), la carga puntual se encuentra corrida del centro de la esfera, por lo cual la distribución resultante cambia. En la pared interior del conductor aparece una carga neta-Q distribuida no homogéneamente, con más concentración de carga negativa en la zona más próxima a la carga puntual y menos concentración a medida que los puntos de la pared interior se encuentren más lejos de la carga. Sin embargo, en la pared exterior de la esfera, la distribución de carga sigue siendo homogénea y con una carga neta $+\mathrm{Q}$. Tal distribución es homogénea porque el campo eléctrico en el interior del conductor tiene que ser cero. Por lo tanto la respuesta sería que el conductor no influye en el campo eléctrico exterior a él generado por la carga puntual 
+ Q ubicada en cualquier punto de la cavidad. En otras palabras, el campo eléctrico fuera del conductor no cambia si cambia la ubicación de la carga $+Q$ en el interior de la cavidad.

Para poder contestar las preguntas, los estudiantes se abocaron a entender cómo son las distribuciones de cargas tanto en la superficie interior de la esfera como en la superficie exterior de la misma. Esta cuestión es la que les dificultó el camino en la búsqueda de una respuesta, puesto que no lograban convencerse de cómo era efectivamente esa distribución. Tal camino de búsqueda dio lugar a una valiosa fuente de información para poder entender sus razonamientos desde una perspectiva epistémica.

a) La unidad de análisis. El trabajo que se presenta es un estudio de caso cuya unidad de análisis es el grupo de los tres estudiantes (M, S y A) mientras resuelven el problema del enunciado 1. La forma en la que se planteó la tarea y la resultante interactividad entre ellos durante la resolución impide observar los razonamientos individuales de cada estudiante. Nunca podríamos tener la certeza sobre si una idea que un estudiante expresa proviene sólo de sus experiencias y conocimientos previos, o si surge como consecuencia de la interacción con sus pares. Por lo tanto, lo que sí sabemos es que son razonamientos que surgen de los estudiantes como consecuencia de la interacción social de varios individuos en un grupo. (MORTIMER, 2005).

Consideramos que el estudio de caso es la metodología de indagación más adecuada para entender la complejidad y la dinámica de la dimensión epistémica de los estudiantes. Entendemos al estudio de caso como un método de indagación que rescata la particularidad y complejidad de un caso singular, para llegar a comprender el fenómeno en circunstancias más globales. Su objetivo básico es comprender una experiencia, dentro de su contexto real. La potencia y justificación investigadora de un estudio de caso se basa en el supuesto de que lo global se refleja en lo local. (ÁLVAREZ ÁLVAREZ; SAN FABIÁN MAROTO, 2012). Aunque la posibilidad de generalización de un caso es escasa, los datos surgidos de éste son un ingrediente esencial para el planteo de futuras hipótesis.

\section{Resultados y análisis ${ }^{3}$}

El presente análisis se realiza sobre registros obtenidos durante la segunda de una serie de entrevistas. Durante la primera entrevista, estos estudiantes no llegaron a una respuesta satisfactoria para ellos. En esta segunda entrevista, que ocurrió aproximadamente un mes luego de la primera, E comenzó planteándoles (nuevamente) el mismo problema del enunciado 1. La discusión sobre este problema se extendió por más de una hora. En este apartado, transcribimos algunos extractos, que hemos considerado los más significativos para nuestros objetivos.

\section{Episodio I: Invocación a la Autoridad es anidado en un enmarque general dominado por Mapeo Físico.}

Los estudiantes están intentando entender cómo debería ser la distribución de cargas en el conductor. Más específicamente, intentan encontrar cómo se habrá modificado la distri-

\footnotetext{
${ }^{3}$ Resultados preliminares fueron presentados en el XII Simposio de Investigación en Educación en Física (SIEF) realizado en Tandil en 2014. (PEREA; BUTELER, 2014). 
bución de cargas en la cara interna del conductor esférico debido al desplazamiento de la carga puntual $\mathbf{Q}$ hacia fuera del centro de la cavidad. Comienzan considerando lo que ocurre en dos puntos en el interior del conductor, y a la misma distancia del centro geométrico, representados por "a" y "b" en el esquema de la figura 1. Su razonamiento sigue la siguiente lógica: El campo en el punto "b" es la suma del campo generado por $\mathbf{Q}$ y el generado por la carga inducida $d q_{2}$ ubicada en el punto 2. De la misma manera, la contribución al campo en el punto "a" es la suma del campo generado por $\mathbf{Q}$ más el generado por la carga inducida $d q_{1}$ ubicada en 1 . Dado que la distancia desde $\mathbf{Q}$ a "b" es mayor que la distancia desde $\mathbf{Q}$ a "a" y que en los dos casos el campo resultante debe ser cero, deducen que la carga inducida $d q_{2}$ debe ser menor que $d q_{1}$.

Figura 1. Esquema explicativo del razonamiento de los estudiantes en el Episodio I

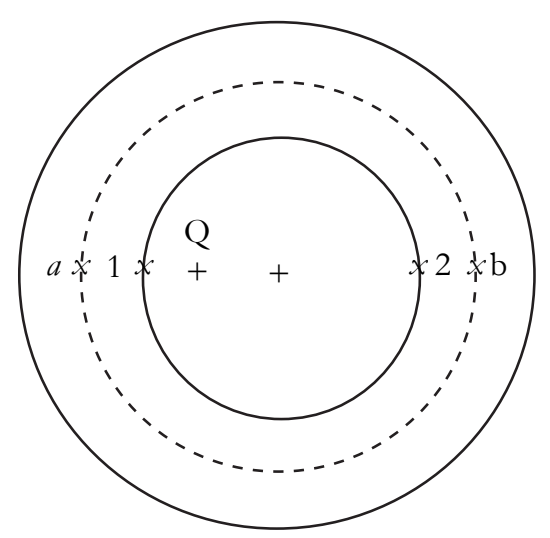

Fuente: elaborado por los autores.

1. M: Porque podemos poner... digamos... como tenés el principio de superposición de la fuerza y que el campo es la fuerza sobre la carga podés sumar, viste, campos así... y podés fijarte ... O sea... la suma del campo generado por eso [se refiere a la carga puntual] más la suma del campo generado por las cargas que están abi [se refiere a las inducidas en la superficie interior de la esfera] tienen que dar cero [para cualquier punto entre los radios interior y exterior de la esfera]. Podés agarrar dos vectores digamos, dos vectores [diametralmente opuestos]... ¡ cualquiera!, dos $\mathrm{r}$ uno que vaya para acá [desde la carga puntual hasta el punto más cercano correspondiente a la pared interior de la esfera] y otro que vaya para allá [desde la carga puntual hasta el punto más lejano correspondiente a la pared interior de la esfera], como uno va a ser más grande que el otro digamos... viendo el dibujo, ehbh... te va a quedar que el campo ejercido acá es más débil que este de acá...

2. A: Si...

3. $\mathbf{M}:$ Y... digamos... si se supone que tiene que haber como una distribución superficial... pero no puede ser... 
4. M: ¿Puede estar adentro? ¿Puede estar adentro... la distribución?

5. A: ¿Cómo?

6. M: La distribución de cargas...

7. A: Y, tiene que estar en la superficie...

8. M: Ah, ¿sí o sí en la superficie?...

9. S: Sí, sí, sino no van a ser cargas... suponemos que no...

10. M: Sí, no, pero decía como fjjarse para ver el tema de cómo va a estar distribuida la carga en la superficie usar el campo, usar los campos, que la suma sea cero y ver con dos vectores, uno que esté más lejos y otro que esté más cerca, dos vectores posición r digamos...

11. A: Claro, o sea, yo no sé por qué solo dos vectores...

12. M: No, para compararlos para que una te dé que el que tiene el vector más largo tiene la distribución menor que el otro... Por eso digo dos ... pero en realidad a eso lo bacés con todos...

13. A: Y lo que podemos hacer es una integral de, podemos hacer una integral... no sé cómo pero podemos intentar...

Este razonamiento se sostiene en consideraciones de tipo geométrico (las relaciones de distancia que se evidencian en el esquema) y algebraico (las contribuciones a los campos crecen con la magnitud de la carga fuente y decrecen con la distancia de la misma al punto en cuestión), por ello interpretamos que en el turno 1 se está enmarcando al problema como Mapeo Físico.

Aún cuando el enmarque es el de mapeo físico, aparecen indicadores de invocación a la autoridad. Las cargas inducidas $\left(d q_{1}\right.$ y $d q_{2}$ en el esquema de la figura 1) deben estar ubicadas en la superficie del conductor. Lo que les permite afirmar esto es la activación de invocación a la autoridad (turnos 4 a 9). Esta afirmación es producida como una regla válida sin cuestionamiento. La línea 9, particularmente, da cuenta de esto: $\mathbf{S}$ trata de responder a la inquietud de sus compañeros sobre si la carga debe "sí o sì" estar en la superficie. Comienza intentando dar razones (porque si no serían cargas...) pero interrumpe ese discurso para simplemente afirmar "suponemos que no". Es decir, puede evitar buscar las razones para afirmar que las cargas están en la superficie y tiene confianza en que la suposición es válida. Invocación a la autoridad se incorpora al enmarque que ya se ha establecido, sin alterar la manera de avanzar en el razonamiento mediante mapeo físico. De este modo, logran articular una lógica de razonamiento sintetizada en dos puntos: (i) como la distancia desde Q a los puntos a y b son distintas, las cargas inducidas en los puntos 1 y 2 también lo son; y (ii) para avalar la afirmación anterior la autoridad provee un elemento crucial: la carga inducida debe estar en la superficie interior (turnos 7 a 10). Es decir, la invocación a la autoridad contribuye con el mapeo físico.

Es importante notar, sin embargo, que invocar a la autoridad, y mapeo físico son dos formas distintas de gestionar el conocimiento que no pueden coexistir simultáneamente. No es posible tratar al conocimiento como algo que se construye (mapeo físico) y al mismo tiempo aceptar un enunciado por el sólo hecho de provenir de una fuente autorizada. Esto nos permite entender que, mientras se invoca a la autoridad, mapeo físico queda momentáneamente suspendido. Al final de este episodio, A plantea la posibilidad de hacer una integral que les permita obtener el resultado buscado, por lo que se advierte que el enmarque vuelve a estar dominado por mapeo físico. Quieren hacer esa integral que les permitirá ajustar mejor la matemática a la descripción física. La sofisticación epistémica de estos estudiantes se ve manifestada entonces por la ma- 
nera en que Invocación a la Autoridad puede interrumpir momentáneamente el enmarque inicial (Mapeo Físico) para proveer una pieza fundamental de información. Invocar a la autoridad se ha anidado en Mapeo Físico. Se trata de una anidación porque Invocar a la Autoridad no se activa como alternativa al enmarque inicial, sino para proporcionar un aporte a la actividad propiciada por ese enmarque.

\section{Episodio II: Un recurso de analogía es anidado en un enmarque general dominado por Mapeo Físico}

Hacia el final del episodio I, vimos a los estudiantes analizar cómo se altera la distribución de cargas inducidas originalmente con simetría esférica en la cara interior del conductor. Al comenzar este episodio, veremos cómo, ante la imposibilidad de llevar a cabo un cálculo, optan por una movida epistémica interesante: plantean un caso análogo, geométricamente más sencillo: el de un anillo de carga y una carga puntual, primero en el centro, y luego descentrada.

El uso de analogías ha sido ya descripto y reportado en la literatura (CLEMENT, 1993; FERRAZ; TERRAZZAN, 2003; MAY; HAMMER; ROY, 2006). May, Hammer y Roy (2006) ofrecen una revisión de los trabajos que se refieren a las analogías, tanto planteadas por textos, por los docentes y por los mismos estudiantes. Los estudios allí revisados ponen de manifiesto la importancia de la utilización de analogías en los procesos de comprensión conceptual, y destacan que la capacidad de proponer y utilizar analogías es una característica fuertemente vinculada a la construcción de conocimiento científico y por ende una característica de la experticia científica. En particular, algunos autores muestran que los estudiantes generan analogías de manera espontánea, evalúan su validez y las refinan en consecuencia, de modo que pueden usarla eficazmente para construir nuevo conocimiento y para comunicar sus ideas a otros. En nuestro análisis, lo que veremos evidenciado en las alocuciones de los estudiantes es un enmarque dominado por el recurso de analogía, definido de la siguiente manera: es válido sacar conclusiones sobre el caso de interés a partir del análisis de otro caso que comparte ciertas características con el primero.

$\mathrm{Al}$ igual que anteriormente, analogía no rompe el enmarque establecido, sino que se incorpora a él. Ellos no abandonan el marco de mapeo físico, sino que incorporan analogía para poder llevar adelante al ajuste con la matemática que aquél enmarque impone. La jugada es exitosa en términos epistémicos, más allá de que no logren el resultado buscado.

Figura 2. Esquema en donde se representa la analogía que los estudiantes están tratando de establecer

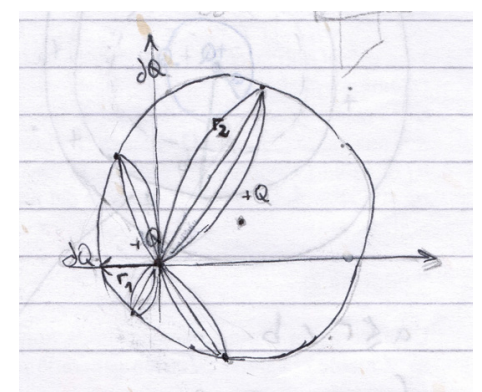

Fuente: elaborado por los estudiantes. 
14. A: ... después de todo, esto es una esfera que tiene la carga acá [se refiere a la superficie interior de la esfera], pero como es simétrica supongo que lo podemos pensar como que es un círculo con cada uno [cada porción diferencial] un delta $q$ de carga y ver de hacer la integral y a los radios los podemos escribir... eso no sé cómo pero podemos ver cómo hacerlo...

15. S: $A$ ver... [S empieza a dibujar la Figura 2, en este caso sólo dibuja un anillo con la carga descentrada y ubica el origen del sistema de coordenadas en la misma] vos tenés una carga $+Q \ldots$

16. A: Sí...

17. S: y vos decís que tenés en este anillo como querés pensar vos...

18. A: Claro, de una...

19. M: Que en total tiene una carga $-Q$

//

20. M: Pero lo que queremos ver es el tema de la distribución de los delta q... [se refiere a cómo se distribuyen las cargas en el anillo].

21. A: Claro...Sí.

22. M: ¿Eso queremos no?

En las líneas 15 y 16, se explicitan dos cosas importantes. En primer lugar, que A propone trabajar sobre un análogo del problema original: un anillo de carga con distribución uniforme. En segundo lugar, explicita que esta analogía le servirá para llevar adelante el cálculo que necesita hacer. Es decir, la analogía aparece como una manera válida de obtener una predicción. Asume que trabajar sobre el análogo le permitirá obtener conclusiones válidas acerca del caso original. También es claro que esta analogía no aparece como alternativa al razonamiento previo, sino como un aporte para continuarlo. Las alocuciones de las líneas 18 a 22 refuerzan esta interpretación.

En las líneas 23 a 33 explicitan cómo un cálculo les permitirá, según el resultado que se obtenga, aceptar o descartar la hipótesis de que la distribución de carga inducida (o la correspondiente carga distribuida sobre el caso análogo, el anillo) es uniforme.

23. M: Igual vos sabés que está en equilibrio [se refiere a una carga puntual que dibujó en la Fig. 4, ubicada en el centro del anillo]. O sea, que la sumatoria de las fuerzas tiene que ser cero. Que podés poner la sumatoria de fuerzas [sobre Q] ... toda la sumatoria de los delta $q$... por ehh... no, sobre el radio al cuadrado. ¿O no? $Y$ el radio al cuadrado es para todos la distancia del radio...

24. A: Si tenés la cosa acá [la carga puntual en el centro]. La suma de todas las fuerzas, que va a ser lo mismo que la suma de los campos, es igual a cero...

25. S: O sea... las cargas por ejemplo... dejás las cargas fjjas acá afuera [distribuidas de manera homogénea]...

26. A: Claro y ahora ver si corriendo eso... si corriendo esta carguita [fuera del centro] ebh obtenés... o sea, esto sigue siendo una sumatoria de fuerzas igual a cero o sumatoria de campos igual a cero si tomo cada delta q acá. Bueno y para eso ¿cómo podemos hacerlo? Bueno, en principio se me ocurre que... tenemos que poner un sistema de coordenadas. 
27. S: Bueno, tenemos $+Q$ acá [dibuja el origen del sistema de coordenadas en donde está la carga Q descentrada como se ve en la Fig. 3]...

28. E: ¿Para qué te va a servir saber que la suma de todas las fuerzas es cero?

29. A: Y porque si es cero entonces las cargas en la superficie interior van a quedar distribuidas homogéneamente y si quedan distribuidas homogéneamente afuera también va a ser igual y ya está...

30. S: ...vamos a ver qué pasa. Suponiendo esto, que están distribuidas uniformemente y vos corrés la carga [fuera del centro], suponemos que siguen [distribuidas] uniformemente, vamos a ver... vamos a llegar a un absurdo...

31. A: $A$ un absurdo o no.

32. S: Sí.

33. A: Ojalá que no lleguemos a un absurdo porque si no... Bueno si llegamos a un absurdo o si llegamos a que la sumatoria de fuerzas es distinta de cero, que... o sea que la sumatoria de campos es distinta de cero, entonces estaríamos llegando a que no va a quedar homogénea...

Entre las líneas 24 y 27 los estudiantes se detienen a analizar una característica importante que observan en el caso de la carga puntual Q ubicada en el centro del anillo conductor cargado: la carga inducida por la presencia de Q, distribuida de manera uniforme sobre el anillo ${ }^{4}$ (simetría circular), es tal que si fueran a considerar el campo generado por esa carga inducida, éste sería cero justamente en el punto donde se ubica Q. Para ello, hacen consideraciones de tipo geométrico y algebraico, comparando distancias y diferenciales de carga. Estos razonamientos evidencian que permanece activado el marco de mapeo físico que había dado lugar al comienzo de todo el análisis.

Entre las líneas 28 y 33, un “razonamiento por el absurdo" les permite decidir cómo interpretarán el eventual resultado de calcular el campo total, generado por esa carga "inducida", en el punto donde se ubica Q.

A lo largo de este episodio, pudimos observar cómo el enmarque general de mapeo físico guió la construcción de conocimiento. Durante un momento, ese enmarque quedó suspendido mientras se establecía una analogía. En este sentido, al igual que anteriormente, entendemos que es imposible que, al mismo tiempo, los estudiantes estén ajustando la matemática disponible a la descripción física de lo que pasa con la esfera, y pasen a pensar en un caso análogo. Por esa razón, decimos que el mapeo físico se "suspende brevemente" mientras se construye el caso análogo, y luego se reanuda para trabajar ese mismo ajuste en el caso análogo, es decir, analogía se anida en mapeo físico.

Al igual que en el Episodio I, notamos una anidación de un enmarque en otro. La (breve) suspensión de Mapeo físico y la activación de analogía es una movida que epistémicamente apunta a contribuir con el razonamiento que los estudiantes vienen realizando. No se trata de una activación aleatoria, sino de una suspensión y activación que apuntan a reforzar la idea de que Mapeo Físico es la respuesta a la pregunta "qué se supone que debo hacer acá".

\footnotetext{
${ }^{4}$ Es la carga inducida en la esfera conductora, que ahora ha sido ubicada en un anillo.
} 
La sofisticación de estos estudiantes consiste en suspender un enmarque por unos instantes, y activar otro para poder luego continuar con el primero.

\section{Episodio III: La comprensión lograda a través de una analogía y una posible comparación con un enunciado proveniente de la autoridad}

Después de haber cambiado la geometría tridimensional esférica por otra bidimensional circular, los cálculos siguen siendo inaccesibles para los estudiantes y no pueden, por esta vía, confirmar o desestimar que la distribución de carga inducida en la cara interior del conductor no es uniforme cuando la carga puntual está descentrada. Al comienzo de este episodio, A "decide" 5 enfocarse en lo que ocurre con la carga inducida en la superficie externa del conductor, aún sin confirmar lo que sucede con la carga inducida en la cara interior. Esta decisión les permite imaginarse una situación análoga que los libera de hacer los cálculos que no pueden llevar a cabo y, finalmente, les permite decidir y argumentar por qué el campo eléctrico externo al conductor no se ve afectado por el desplazamiento de la carga puntual en su cavidad.

Figura 3. Analogía planteada por los estudiantes

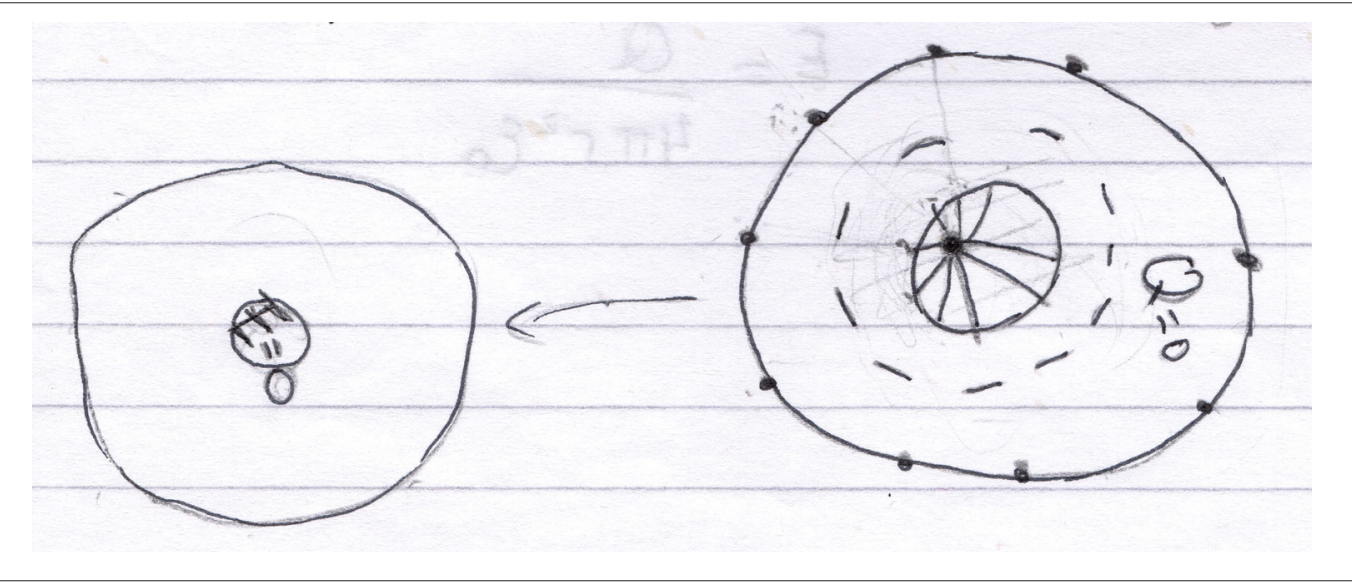

Fuente: elaborado por los estudiantes.

34. A: Sí, yo creo que... que... o sea... lo de adentro [distribución en la cara interior], no sé ya no estoy seguro, no me convenzo del todo pero ponele que se redistribuya un poco más de este lado... creo que eso no va a afectar afuera...

35. M: Bueno, abí está, perfecto, y entonces ¿̇por qué tendría que estar afuera homogénea si esto [en la cara interna] está más para un lado [inhomogénea]?

\footnotetext{
${ }^{5}$ Las comillas indican que se trata de una decisión tomada, muy probablemente, de manera implícita. Esta "decisión" indica la activación de un enmarque para la situación.
} 
36. A: No no pero suponete... no no, suponete que adentro es cero la carga porque adentro de un conductor la carga es cero [refiriéndose al volumen dentro del conductor], si la carga entonces...

37. M: Abh ¿estás haciendo Gauss digamos?

38. A: Claro, una especie de Gauss. En ese sentido... mirá, ponele... acá adentro sabemos que acá el campo y la carga van a ser cero [en referencia a una superficie esférica imaginaria, concéntrica con la esfera, contenida en el interior del conductor y que encierra a la cavidad], [ver lado derecho en líneas de puntos, figura 3].

39. M: Sí.

Vamos a detenernos en el análisis de las intervenciones de $\mathbf{A}$ en las líneas 36 a 39. Si bien utiliza la palabra "suponete", en realidad está invocando a la autoridad: "suponete que la carga es cero porque adentro de un conductor la carga es cero". M y A están intentando ajustar el teorema de Gauss a la situación física que quieren entender: están pensando en una superficie imaginaria (la cual dibujan con líneas de punto, lado derecho de la figura 3). La utilización del teorema de Gauss, con las consideraciones geométricas que ello impone, nos permite interpretar a esta decisión epistémica como un enmarque dominado por mapeo físico. Aún así, ellos pueden suspender brevemente ese enmarque para activar Invocación a la Autoridad. La anidación de invocación a la autoridad permite incorporar una pieza fundamental de conocimiento, desde una fuente autorizada, para ser usada en el proceso de ajustar la geometría y la física. Esto se contrasta con una simple flexibilidad.

40. A: Está... lo estoy repartiendo... pero abhbh no, no está simétricamente [advierte la falta de simetría de las cargas encerradas]. Bueno, no importa, no hagamos Gauss. Sabemos que el campo [en el interior del conductor] es cero adentro... Si el campo es cero creo que la carga va a tener que ser cero. Bueno, ponele, si el campo y la carga [dentro de la superficie "Gaussiana"] son cero, entonces es lo mismo que pensar que acá [señala la superficie imaginaria] hay nada de carga... que tiene un bueco nada más... ¿isería lo mismo? A mi se me ocurre que sí, con las cargas acá [en la superficie exterior] repartidas de algún modo, si acá se puede considerar que acá $Q=0$ nomás...

A establece algunas cuestiones importantes:

i) La superficie imaginaria que dibujó (parte derecha de la figura 6) no es una "buena" candidata para aplicar el Teorema de Gauss, dada la imposibilidad de establecer con certeza la simetría esférica del campo eléctrico.

ii) Sí puede afirmar que el campo eléctrico sobre la superficie imaginaria es cero (esa superficie está siempre dentro del conductor) y que la carga neta encerrada es igual a cero.

iii) Las consecuencias de las dos afirmaciones anteriores le permiten establecer una analogía que le ayudará a resolver el problema 
41. M: Bueno, pero ¿cómo se inducen estas cargas acá [superficie exterior, lado derecho, figura 6] ¿Homogéneas?

42. A: Claro, y si yo puedo pensar que acá [en el interior de la esfera imaginaria] es todo $Q$ igual a cero... es lo mismo...

43. M: Si estuvieran distribuidas homogéneamente sería como tener una carga $Q$ en el centro, entonces el caso de que ésta [la carga puntual de la esfera de la derecha de la Fig. 6] esté en el medio ¿sería lo mismo que eso?

44. A: Para afuera sí.

45. A: ¿Cómo era el dicho... no, no era un dicho, pero cómo era lo que te habían dicho? ¿Era que 'las de adentro no se enteran quépasa afuera y las de afuera no se enteran qué pasa adentro', era?

A establece dos analogías encadenadas. En primer lugar, el caso de interés (esfera conductora con la carga puntual en la cavidad, corrida del centro) es equivalente al caso de una esfera en la cual hay una cavidad con "nada de carga", y una carga neta igual a +Q distribuida homogéneamente sobre la superficie exterior. Es decir, advierte que, desde la superficie imaginaria hacia fuera, todo se "ve" igual que si se reemplazara esa superficie por una esfera con "nada de carga". En segundo lugar, una esfera con "nada de carga" en la cavidad correspondiente a la superficie imaginaria y una carga neta $+Q$ distribuida homogéneamente en la superficie exterior, es equivalente (para el exterior de la esfera) a un conductor descargado con una cavidad en la cual se ha colocado una carga puntual $+Q$ en su centro. De este modo, logra establecer que la carga descentrada generará, hacia fuera de la superficie exterior del conductor, la misma configuración de campo eléctrico que si estuviera ubicada en el centro de la cavidad (ver figura 4).

Figura 4. Cadena de analogías elaborada por los estudiantes

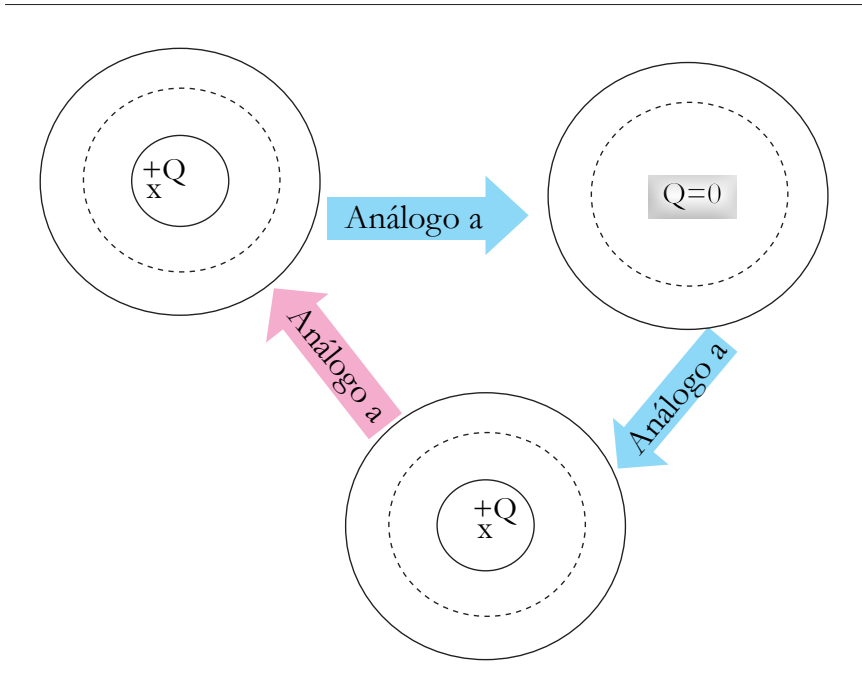

Fuente: elaborado por los autores. 
En el turno 45, A invoca a la autoridad. En primer lugar, intenta recuperar un enunciado como un "dicho". Se corrige, para decir "lo que te habian dicho". Hacia el final, quiere corroborar el enunciado, preguntando “ ¿era?". Es decir, la veracidad del enunciado radica en la exactitud con que puedan reproducirlo. Nuestro conocimiento sobre las características del curso en la carrera de Licenciatura nos permite reconocer que ese "dicho" es la manera usual de referirse a las propiedades eléctricas de una jaula de Faraday.

Esta última alocución puede entenderse como un indicio de que, luego de haber obtenido una conclusión satisfactoria, en un enmarque dominado por el recurso de mapeo físico, en el cual, además, se han anidado invocación a la autoridad y mapeo físico, los estudiantes están intentando articular ese razonamiento con un enunciado proveniente de la autoridad. En este caso, se trata sólo de un atisbo de esa intención y no avanzan en esa comparación. De hecho, es la primera vez en toda la entrevista que estos estudiantes tienen la posibilidad de comparar conclusiones obtenidas según dos marcos epistémicos diferentes.

\section{Consideraciones finales}

El análisis de esta entrevista, estructurado en tres episodios, ha permitido encontrar diferentes elementos que constituyen, a nuestro criterio, un aporte a la idea de sofisticación epistémica en el contexto de la descripción de las epistemologías personales. Este aporte viene dado en dos ejes:

\section{La cuestión de la coexistencia de enmarques y el refinamiento de la idea de anidación}

Bing y Redish (2012) proponen la idea de anidación. Durante el proceso de análisis que llevamos a cabo durante este trabajo, encontramos una sutil característica en esta idea de anidación que no había sido discutida por los autores que la propusieron. Nos preguntamos si los enmarques anidados pueden coexistir con el enmarque en el que se anidan.

En un marco epistémico son activados un conjunto de recursos. Un subconjunto de ellos son recursos epistémicos que justamente dan sentido a la tarea que el sujeto realiza, o como ya se ha mencionado, permiten dar respuesta a la pregunta implícita "qué se supone que debo hacer acá". En este sentido, es posible imaginar, por ejemplo, que en un marco epistémico dominado por el recurso de dar sentido ${ }^{6}$, pueda estar activado mapeo físico. Ajustar la matemática y el comportamiento físico de un sistema es, en sí misma, una manera de dar sentido a lo que uno entiende. En este caso, entendemos, no se trataría de la anidación de mapeo físico en el enmarque de dar sentido, sino simplemente en que ese enmarque inicial se ha enriquecido. Sin embargo,

\footnotetext{
${ }^{6}$ El recurso de dar sentido, descripto por ejemplo en Hutchison y Hammer (2010), permite al sujeto tratar al conocimiento como algo que debe construir de manera que tenga sentido para él/ella a partir de piezas de información que pueden provenir de distintas fuentes, que pueden abarcar la experiencia cotidiana, la instrucción previa, la autoridad, la ocurrencia, etc.
} 
en otros casos, es posible advertir que en un enmarque inicial X, se anida otro enmarque $\mathrm{Y}$, el cual, en principio, resulta incompatible con el primero. Por ejemplo:

i) En el Episodio I los estudiantes llevan a cabo un razonamiento sostenido por mapeo físico y en un momento (turnos 4 a 9) invocan a la autoridad, para recuperar la afirmación de que las cargas deben estar en la superficie del conductor. Un sujeto que está intentando ajustar la matemática a una situación física en particular (mapeo físico) está construyendo conocimiento en ese momento. Esta postura constructivista en relación al conocimiento se contrapone con una postura transmisionista en la cual el conocimiento es tratado como información que se recupera desde una fuente autorizada. En otras palabras, el estudiante no podría, simultáneamente, tener dos posturas contrapuestas frente al conocimiento: constructivista y transmisionista. Por esta razón, estamos en condiciones de afirmar que mientras se activa invocar a la autoridad, mapeo físico queda momentáneamente suspendido.

ii) En el Episodio II, los estudiantes llevan adelante un razonamiento que está propiciado a partir de la activación de mapeo físico. En esas circunstancias, la imposibilidad de realizar un cálculo les impide llevar adelante el ajuste que necesitan hacer. Es entonces cuando activan analogía. Esto les permite "cambiar" la esfera por un anillo y trabajar sobre esa nueva situación. Es imposible que los estudiantes construyan conocimiento haciendo un mapeo físico en un conductor esférico, y, simultáneamente, lo hagan mediante el análisis de la distribución de cargas en un anillo.

Por estas razones, creemos estar en condiciones de afirmar que la sofisticación epistémica de estos sujetos no consiste en la coexistencia de distintos enmarques sino en la activación sucesiva de los mismos. Dado un enmarque $\boldsymbol{X}$, anidar es la posibilidad de suspenderlo brevemente, activar otro $\boldsymbol{Y}$ por un instante, superar algún obstáculo, y retomar el enmarque inicial para continuar.

Esta idea más elaborada de anidación nos permitió advertir que los sujetos que participan de nuestro estudio muestran un grado de sofisticación epistémica diferente de los reportados por Bing y Redish (2012), lo cual constituye el otro aspecto del aporte logrado en este trabajo.

\section{Un nuevo grado de sofisticación epistémica}

Bing y Redish (2012) reportan tres grados distintos de sofisticación epistémica. En primer lugar, y como evidencia de nula sofisticación, casos en que los estudiantes no pueden cambiar de marco epistémico. Un primer grado de sofisticación es el de flexibilidad epistémica, entendida como la posibilidad de enmarcar la tarea de más de una manera. El grado superior de sofisticación que describen, es aquel en el cual los sujetos, además de evidenciar flexibilidad, anidan distintos enmarques en un enmarque general que los autores denominan de "valoración de coherencia", según el cual se intenta encontrar la coherencia entre razonamientos llevados a cabo en diferentes enmarques.

La idea de anidación refinada en la sección anterior nos permitió advertir una cuestión central para nuestro análisis. Nos permitió diferenciar a nuestros sujetos de los sujetos que muestran un comportamiento epistémicamente flexible, entendiendo la flexibilidad como la mera capacidad de enmarcar la misma situación de maneras diferentes. Es decir, nos permitió advertir que estábamos ante un grado de sofisticación mayor que la mera flexibilidad. Nues- 
tros sujetos, no sólo pueden activar diferentes enmarques, sino que pueden anidar esos nuevos enmarques en los ya establecidos. Esas anidaciones les proporcionan una manera de superar situaciones que por momentos parecen callejones sin salida. A partir de un enmarque inicial, y frente a obstáculos para avanzar en el razonamiento, pueden anidar nuevos enmarques que colaboran en el escenario general de indagación y construcción de conocimiento establecida por el enmarque inicial. Aun así, la sofisticación aquí reportada no muestra aquella componente metacognitiva tan clara que muestra el sujeto $\mathrm{S}_{3}$ en el trabajo de Bing y Redish (2012), quien puede comparar caminos de pensamiento diferentes en un marco general de valoración de la coherencia. Creemos, sí, que estamos ante la presencia de un estadio intermedio entre la mera flexibilidad y la valoración de coherencia. Es plausible que los sujetos de nuestro estudio estuvieran en camino a ese grado de mayor sofisticación y en un futuro lograsen evidenciar esa conducta metacognitivamente más rica. De hecho, ellos hacen un atisbo (final del Episodio III) de relacionar una información proveniente de la autoridad con un resultado que obtuvieron luego de trabajar arduamente con analogías y mapeo físico.

Nos parece importante poder distinguir y comenzar a caracterizar estos estados intermedios que indican que el sujeto está avanzando en su aprendizaje epistémico, dado que esta es una componente del aprendizaje de la física que no ocupa un lugar explícito en la agenda de enseñanza.

\section{Referencias}

ÁLVAREZ ÁLVAREZ, C; SAN FABIÁN MAROTO, J. L. La elección del estudio de caso en investigación educativa. Gazeta de Antropología, Granada, v. 28 n. 1, 2012. Disponible en: <http://hdl.handle.net/10481/20644>. Visitado en: 11 enero 2017.

BING, T. J.; REDISH, E. F. Analyzing solving using math in physics: epistemological framing via warrants. Physical Review: physics education research, College Park, v. 5, n. 2, p. 020108, 2009. Disponible en: <http://journals.aps.org/prper/pdf/10.1103/ PhysRevSTPER.5.020108>. Visitado en: 11 enero 2017.

. Epistemic complexity and the journeyman-expert transition. Physical Review: physics education research, College Park, v. 8, n. 1, p. 010105, 2012. Disponible en: <https:// doi.org/10.1103/PhysRevSTPER.8.010105>. Visitado en: 11 enero 2017.

CAMPANARIO, J. M.; OTERO, J. C. Más allá de las ideas previas como dificultades en el aprendizaje: las pautas de pensamiento, las concepciones epistemológicas y las estrategias metacognitivas de los alumnos de ciencias. Enseñanza de las Ciencias, Barcelona, v. 18 n. 2, p. 154-169, 2000. Disponible en: < https://ddd.uab.cat/pub/ edlc/02124521v18n2/02124521v18n2p155.pdf>. Visitado en: 11 enero 2017.

CLEMENT, J. Using bridging analogies and anchoring intuitions to deal with students' preconceptions in physics. Journal of Research in Science Teaching, Hoboken, v. 30 n. 10, p. 1241-1257, 1993. Disponible en: < https://doi.org/10.1002/ tea.3660301007>. Visitado en: 11 enero 2017. 
COLEONI, E. A.; BUTELER, L. M.; PEREA, M. A. Analizing high school students' problem solving through their personal epistemologies. Latin-American Journal of Physics Education, Mexico, v. 8, n. 1, p. 3-12, 2014.

DISESSA, A. A. Toward an epistemology of physics. Cognition and Instruction, Philadelphia, v. 10, n. 2-3, p. 105-225, 1993. Disponible en: <http://www.jstor.org/ stable/3233725>. Visitado en: 11 enero 2017.

ELBY, A.; HAMMER, D. On the substance of a sophisticated epistemology. Science Education, Hoboken, v. 85, n. 5, p. 554-567, 2001. Disponible en: <https://doi. org/10.1002/sce.1023>. Visitado en: 11 enero 2017.

FERRAZ, D. F.; TERRAZZAN, E. A. Uso espontâneo de analogias por professores de biologia e o uso sistematizado de analogias: que relação? Ciência $\boldsymbol{\&}$ Educação, Bauru, v. 9, n. 2, p. 213-227, 2003. Disponible en: < https://doi.org/10.1590/S1516$73132003000200005>$. Visitado en: 11 enero 2017.

GURIDI V. ¿Puede vincularse la comprensión conceptual en física con el perfil epistemológico de un estudiante? 1999. Tesis (Maestría en Epistemología y Metodología de la Ciencia) - Universidad Nacional de Mar del Plata, Mar del Plata, 1999.

HAMMER, D. The variability of student reasoning, lecture 3: manifold cognitive resources. In: REDISH, E.; VICENTINI, M. (Ed.). Research on physics education. Bologna: Società Italiana di Física, 2004. p 321-340. (Proceedings of the International School of Physics "Enrico Fermi”, 156).

HAMMER, D.; ELBY, A. On the form of a personal epistemology. In: HOFER, B. K.; PRINTRICH, P. R. (Ed.) Personal epistemology: the psychology of beliefs about knowledge and knowing. Mahwah: Lawrence Erlbaum, 2002. p. 169-190.

HAMMER, D. et al. Resources, framing, and transfer. In: MESTRE, J. (Ed.). Transfer of learning from a modern multidisciplinary perspective. Greenwich: Information Age Publishing, 2005. p. 89-119.

HOGAN, K. Relating students' personal frameworks for science learning to their cognition in collaborative contexts. Science Education, Hoboken, v. 83, n. 1, p. 1-32, 1999. Disponible en: <https://doi.org/10.1002/(SICI)1098-237X(199901)83:1<1::AIDSCE1>3.0.CO;2-D>. Visitado en: 11 enero 2017.

HUTCHISON, P.; HAMMER, D. Attending to student epistemological framing in a science classroom. Science Education, Hoboken, v. 94, n. 3, p. 506-524, 2010. Disponible en: <https://doi.org/10.1002/sce.20373>. Visitado en: 11 enero 2017.

LISING, L.; ELBY, A. The impact of epistemology on learning: a case study from introductory physics. American Journal of Physics, Melville, v. 73, n. 4, p. 372-82, 2005. Disponible en: <https://doi.org/10.1119/1.1848115>. Visitado en: 11 enero 2017. 
LOUCA, L. et al. Epistemological resources: applying a new epistemological framework to science instruction. Educational Psychologist, Philadelphia, v. 39, n. 1, p. 57-68, 2004. Disponible en: <https://doi.org/10.1207/s15326985ep3901_6>. Visitado en: 11 enero 2017. MAY, D. B.; HAMMER, D.; ROY, P. Children's analogical reasoning in a third-grade science discussion. Science Education, Hoboken, v. 90, n. 2, p. 316-330, 2006. Disponible en: <https://doi.org/10.1002/sce.20116>. Visitado en: 11 enero 2017.

MORTIMER, E. F. Lenguaje y formación de conceptos en la enseñanza de las ciencias. Madrid: Antonio Machado Libros, 2005.

PEREA, M. A.; BUTELER, L. M. Sofisticación epistémica en electrostática: un estudio de caso en alumnos universitarios. Revista de Enseñanza de la Física, Córdoba, v. 26, n. extra, 185-193, 2014. Disponible en: < https://revistas.unc.edu.ar/index.php/revistaEF/ article/view/9749/10487>. Visitado en: 11 enero 2017.

REDISH, E. A theoretical framework for physics education research: modeling student thinking. In: REDISH, E.; VICENTINI, M. (Ed.). Research on physics education. Bologna: Società Italiana di Física, 2004. Bologna: Italian Physical Society, 2004. p.1-56. (Proceedings of the International School of Physics "Enrico Fermi", 156).

RESNICK, R.; HALLIDAY, D.; KRANE, K. Física. 4. ed. México: Continental, 1993. v. 2.

ROSENBERG, S.; HAMMER, D.; PHELAN, J. Multiple epistemological coherences in an eighth-grade discussion of the rock cycle. Journal of the Learning Sciences, Philadelphia, v. 15, n. 2, p. 261-292, 2006. Disponible en: < https://doi.org/10.1207/ s15327809jls1502_4>. Visitado en: 11 enero 2017.

SANDOVAL, W. A. Understanding students' practical epistemologies and their influence on learning through inquiry. Science Education, Hoboken, v. 89, n. 4, p. 634-656, 2005. Disponible en: <https://doi.org/10.1002/sce.20065>. Visitado en: 11 enero 2017.

SERWAY, R. Física: tomo II. 4. ed. México: McGraw-Hill, 1997.

WAINMAIER, C. Incomprensiones en el aprendizaje de la mecánica clásica básica. 2003. Tesis (Maestría en Enseñanza de las Ciencias) - Facultad de Ciencias Exactas y Tecnología, Universidad Nacional de Tucumán, Tucumán, 2003.

WAINMAIER, C.; SPELTINI, C.; SALINAS, J. Conceptos y relaciones entre conceptos de la mecánica newtoniana en estudiantes que ingresan a la universidad. Revista Electrónica de Enseñanza de las Ciencias, Vigo, v. 10, n. 1, p. 133-152, 2011. Disponible en: < http:// reec.uvigo.es/volumenes/volumen10/ART7_Vol10_N1.pdf>. Visitado en: 11 enero 2017.

Artigo recebido em 16/11/2015. Aceito em 13/08/2016.

Dirección para contacto: Medina Allende y Haya de la Torre, Ciudad Universitaria, Córdoba, 5000, Argentina. 